\title{
HOLOMORPHIC SECTIONAL CURVATURES OF BOUNDED HOMOGENEOUS DOMAINS AND RELATED QUESTIONS
}

BY

J. E. D'ATRI

\begin{abstract}
This paper considers a class of homogeneous Kähler metrics which include the Bergman metrics on homogeneous bounded domains. We obtain various necessary conditions for (a) nonpositive holomorphic sectional curvature, (b) nonpositive sectional curvature, and (c) covariant constant curvature (symmetric metric). In particular, we give examples showing that there exist homogeneous bounded domains which in the Bergman metric have some positive holomorphic sectional curvature.
\end{abstract}

1. Introduction. We will consider a class of homogeneous Kähler metrics on which a solvable group acts simply transitively as a group of holomorphic isometries. These metrics are defined in terms of admissible linear forms on normal $j$-algebras. This class strictly contains the class of Bergman metrics on homogeneous Siegel domains (equivalently homogeneous bounded domains). As general references on homogeneous Siegel domains and their relationship to normal $j$-algebras we list [8], [9] and [10]; some important results are also given in [3], [7] and [11] and some of this paper uses results given in [2].

Defintion. Let $\xi$ be a real (finite-dimensional) Lie algebra and $j$ a vector space endomorphism of $\mathfrak{\xi}$. Then $(\xi, j)$ is called a normal $j$-algebra if there exists a linear form $\omega$ on $\mathfrak{g}$ (called admissible) such that for all $X, Y$ in $\mathfrak{s}$

(D1) $j$ is an almost complex structure, i.e. $j^{2} X=-X$,

(D2) $[X, Y]+j[j X, Y]+j[X, j Y]=[j X, j Y]$,

(D3) $\omega[j X, j Y]=\omega[X, Y]$,

(D4) $\omega[j X, X]>0$ for $X \neq 0$,

(D5) $\Xi$ is solvable.

As observed by Rossi and Vergne [9], [10], these conditions imply that the eigenvalues of the adjoint representation of $\xi$ are real so this agrees with the definition of Pyatetskii-Shapiro [8].

Let $(\xi, j)$ be a normal $j$-algebra with admissible form $\omega$ and $S$ a connected Lie group with Lie algebra 3 . The bilinear form $\langle X, Y\rangle=\omega[j X, Y]$ on $\Xi$ determines a left-invariant metric, also denoted $\langle$, $\rangle$, on $S$. Further, $j$ gives an integrable left-invariant almost complex structure on $S$ with respect to which $\langle$,$\rangle is a Kähler metric. We will be studying metrics which, up to holomor-$

Received by the editors December 14, 1978.

AMS (MOS) subject classifications (1970). Primary 32M10, 53C30.

() 1979 American Mathematical Society 
phic isometry, can be obtained in this way. By results of [3] and [6] (see [7, pp. 47, 51]) the Bergman metrics on bounded homogeneous domains are of this type while by results of [1, pp. 325, 327] (cf. also results of Heintze and Wolf quoted in [1]), [4], and [6], the same is true for any homogeneous, nonflat, Kähler-Einstein metric with nonpositive sectional curvature (whose underlying manifold is automatically simply connected by [5]).

Now fix a normal $j$-algebra $(\xi, j)$ and an admissible form $\omega$ giving the inner product $\langle X, Y\rangle=\omega[j X, Y]$. We must recall the basic structure theorem of Pyatetskii-Shapiro [8]; see also [9], [10]. The last conclusion was derived in [2].

StruCtURE ThEOREM. Let $\mathfrak{n}=[\xi, 3]$ and let $a$ be the orthogonal complement of $\mathrm{n}$ in 3 . Then

(T1) $a$ is a commutative subalgebra and $\mathfrak{n}$ can be represented as the direct sum of the root spaces $\mathfrak{n}_{\alpha}=\{X \in \mathfrak{n}:[H, X]=\alpha(H) X, H \in \mathfrak{a}\}$ of the adjoint action of $a$ on $n$.

(T2) Let $\varepsilon_{1}, \ldots, \varepsilon_{R}$ be the roots whose root spaces are mapped into a by $j$. Then $R=\operatorname{dim} a$, the roots $\varepsilon_{1}, \ldots, \varepsilon_{R}$ are linearly independent and, with proper labelling, all roots are of the form $\frac{1}{2} \varepsilon_{k}, \varepsilon_{k}, 1<k<R ; \frac{1}{2}\left(\varepsilon_{m} \pm \varepsilon_{n}\right), 1<m<n$ $<R$ (although not all these need be roots).

(T3) $j n_{e_{k} / 2}=n_{\varepsilon_{k} / 2} j \mathfrak{n}_{\left(e_{m}+\varepsilon_{n}\right) / 2}=\mathfrak{n}_{\left(e_{m}+\varepsilon_{n}\right) / 2}$.

(T4) The root spaces are pairwise orthogonal.

As usual, for each linear functional $\alpha$ on $a$, let $H_{\alpha} \in a$ be defined by $\left\langle H_{\alpha}, H\right\rangle=\alpha(H), H \in a$. Each $\mathfrak{n}_{\varepsilon_{k}}$ is one-dimensional and we will fix $X_{k} \in$ $n_{e_{k}}$ such that $\varepsilon_{l}\left(j X_{k}\right)=\delta_{k l}, k, l=1, \ldots, R$.

Now let $\omega^{\prime}$ be another admissible form giving the inner product $\langle X, Y\rangle^{\prime}=$ $\omega^{\prime}[j X, Y]$. Note that

$$
\begin{aligned}
& {\left[\mathfrak{n}_{\varepsilon_{l}}, \mathfrak{n}_{\alpha}\right]=0 \text { if } \alpha \neq \frac{1}{2}\left(\varepsilon_{k}-\varepsilon_{l}\right) \text { for some } k<l,} \\
& {\left[\mathfrak{n}_{\varepsilon_{l}}, \mathfrak{n}_{\frac{1}{2}\left(\varepsilon_{k}-\varepsilon_{l}\right)}\right] \subset \mathfrak{n}_{\frac{1}{2}\left(\varepsilon_{k}+\varepsilon_{l}\right)} .}
\end{aligned}
$$

From (D3), we find for any $Y \in \mathfrak{n}_{\left(e_{k}-\varepsilon_{1}\right) / 2}$,

$$
0=\omega^{\prime}\left[X_{k}, Y\right]=\omega^{\prime}\left[j X_{k}, j Y\right]=\frac{1}{2} \omega^{\prime}(j Y)
$$

so

$$
\omega^{\prime}\left(n_{\frac{1}{2}\left(\varepsilon_{k}+\varepsilon_{1}\right)}\right)=0
$$

Then, for any $l$ and any root $\alpha$, we get from (1) and (2)

$$
\left\langle j X_{l}, \mathfrak{n}_{\alpha}\right\rangle^{\prime}=-\omega^{\prime}\left[X_{l}, \mathfrak{n}_{\alpha}\right]=0
$$

so $\langle a, n\rangle^{\prime}=0$. Thus, for any admissible form $\omega^{\prime}$, $a$ is still the orthogonal complement of $\mathfrak{n}=[\xi, \mathfrak{\xi}]$. In other words, a (and hence the root space decomposition and the $X_{k}$ ) is completely determined by the structure of the normal $j$-algebra and does not depend on the choice of admissible form. Note 
also that for any admissible $\omega$, the inner product depends only on the values of $\omega\left(X_{k}\right), k=1, \ldots, R$ and

(3) $\left\langle X_{k}, X_{k}\right\rangle=\omega\left(X_{k}\right)$,

(4) $H_{e_{k}}=j X_{k} / \omega\left(X_{k}\right)$,

(5) $\left\langle H_{e_{k}}, H_{e_{k}}\right\rangle=1 / \omega\left(X_{k}\right)$.

2. Curvature formulas. $S$ will denote a connected Lie group whose Lie algebra $\Xi$ is a normal $j$-algebra with admissible form $\omega$ and other notation as in $\$ 1$. With respect to the left-invariant Riemannian metric $\langle$,$\rangle on S$, the Levi-Civita connection $\nabla$ is computed by

$$
2\left\langle\nabla_{X} Y, Z\right\rangle=\langle[X, Y], Z\rangle+\langle[Z, X], Y\rangle+\langle[Z, Y], X\rangle
$$

for $X, Y, Z$ in $\Xi$. It is sometimes convenient to write this as

$$
\nabla_{X} Y=\frac{1}{2}\left\{[X, Y]-(\operatorname{ad} X)^{t} Y-(\operatorname{ad} Y)^{t} X\right\}
$$

where $(\operatorname{ad} X)^{t}$ is the transpose of ad $X$ with respect to $\langle$,$\rangle . For X, Y$ in $\xi$, note that $\nabla_{X}$ is a skew symmetric linear transformation on $\xi$, that $\nabla_{X} Y-$ $\nabla_{Y} X=[X, Y]$, and that $\nabla_{X}(j Y)=j \nabla_{X} Y$ (from the Kähler condition).

After some computation, one finds that

(8) $\nabla_{H}=0$ for $H \in a$,

(9) $\nabla_{Y} Y=\langle Y, Y\rangle H_{\alpha}$ for $Y \in \mathfrak{n}_{\alpha}$,

(10) $\nabla_{Y} H=-\alpha(H) Y$ for $Y \in \mathfrak{n}_{\alpha}$

(11) $\nabla_{X_{k}} X_{l}=\delta_{k l} \omega\left(X_{k}\right) H_{e_{k}}$.

In computing sectional curvatures, we use

$$
\langle R(Y, Z) Z, Y\rangle=-\left\langle\nabla_{Y} Y, \nabla_{Z} Z\right\rangle+\left\langle\nabla_{Y} Z, \nabla_{Z} Y\right\rangle-\left\langle\nabla_{[Y, Z} Z, Y\right\rangle
$$

which holds for any $Y, Z$ in $\xi$.

Suppose now that $k<l$ and $\frac{1}{2}\left(\varepsilon_{k}+\varepsilon_{l}\right)$ is a root. Take $Y \in \mathfrak{n}_{\left(e_{k}+\varepsilon_{l}\right) / 2}$. Then $[Y, j Y] \in \mathrm{n}_{e_{k}}$ so $[Y, j Y]=r X_{k}$ and $-\langle Y, Y\rangle=\omega[Y, j Y]=r \omega\left(X_{k}\right)$ so

$$
[Y, j Y]=-\left(\langle Y, Y\rangle / \omega\left(X_{k}\right)\right) X_{k} \text {. }
$$

Then

$$
\begin{aligned}
& \left\langle\nabla_{Y} Y, \nabla_{j Y} j Y\right\rangle=\langle Y, Y\rangle\langle j Y, j Y\rangle\left\langle H_{\frac{1}{2}\left(e_{k}+e_{1}\right)}, H_{\frac{1}{2}\left(e_{k}-\varepsilon_{1}\right)}\right\rangle \\
& =\frac{1}{4}\langle Y, Y\rangle^{2}\left\{\left\langle H_{e_{k}}, H_{\varepsilon_{k}}\right\rangle-\left\langle H_{\varepsilon}, H_{\varepsilon_{1}}\right\rangle\right\} \\
& =\frac{1}{4}\langle Y, Y\rangle^{2}\left\{\frac{1}{\omega\left(X_{k}\right)}-\frac{1}{\omega\left(X_{l}\right)}\right\} \text {, } \\
& \left\langle\nabla_{\boldsymbol{Y}} \boldsymbol{j} Y, \nabla_{j Y} Y\right\rangle=-\left\langle\nabla_{\boldsymbol{Y}} \boldsymbol{Y}, \boldsymbol{\nabla}_{j \mathbf{j}} \boldsymbol{} Y\right\rangle \text {, } \\
& \left\langle\nabla_{[Y, j Y} j Y, Y\right\rangle=-\left(\langle Y, Y\rangle / \omega\left(X_{k}\right)\right)\left\langle\nabla_{X_{k}} j Y, Y\right\rangle \\
& =-\frac{\langle Y, Y\rangle}{2 \omega\left(X_{k}\right)}\left\{\left\langle\left[X_{k}, j Y\right], Y\right\rangle+\left\langle\left[Y, X_{k}\right], j Y\right\rangle+\left\langle[Y, j Y], X_{k}\right\rangle\right\} \\
& =\langle Y, Y\rangle^{2} / 2 \omega\left(X_{k}\right) \text {. }
\end{aligned}
$$


Thus the holomorphic sectional curvature determined by $Y \wedge j Y$ is given by

$$
\langle R(Y, j Y) j Y, Y\rangle=-\frac{1}{2}\langle Y, Y\rangle^{2}\left\{2 / \omega\left(X_{k}\right)-1 / \omega\left(X_{l}\right)\right\}
$$

and we have proved the following theorem.

THEOREM 1. Suppose $\frac{1}{2}\left(\varepsilon_{k}+\varepsilon_{l}\right)$ is a root for some pair of indices $k<l$ and that all holomorphic sectional curvatures of $S,\langle$,$\rangle are negative (resp.,$ nonpositive). Then $\omega\left(X_{k}\right)<2 \omega\left(X_{l}\right)\left(\right.$ resp., $\left.\omega\left(X_{k}\right)<2 \omega\left(X_{l}\right)\right)$.

Now we will give an analogous result for general sectional curvatures.

THEOREM 2. Suppose $\frac{1}{2}\left(\varepsilon_{k}+\varepsilon_{l}\right)$ is a root for some pair of indices $k<l$ and that all sectional curvatures of $S,\langle$,$\rangle are nompositive. Then \omega\left(X_{k}\right)<\omega\left(X_{l}\right)$.

Proof. If the dimension of the root space exceeds one, we can choose nonzero $Y \in n_{\left(e_{k}+\varepsilon_{1}\right) / 2}, Z \in \mathfrak{n}_{\left(e_{k}+\varepsilon_{1}\right) / 2}$ with $\langle Y, j Z\rangle=0$ and then a computation like the previous shows

$$
\langle R(Y, Z) Z, Y\rangle=-\frac{1}{4}\langle Y, Y\rangle\langle Z ; Z\rangle\left\{1 / \omega\left(X_{k}\right)-1 / \omega\left(X_{l}\right)\right\}
$$

which proves the theorem in this case. However, to give a general proof, it seems necessary to choose entirely different "test vectors."

Fix a unit vector $Z$ in $n_{\left(\varepsilon_{k}-\varepsilon_{1}\right) / 2}$ and let

$$
U=a j X_{k}+b j X_{l}+j Z, \quad V=a X_{k}+\beta X_{l}+Z .
$$

In addition to the covariant derivatives immediately computable from (8)-(11), we need the following:

$$
\begin{aligned}
& {\left[X_{k}, Z\right]=0 \text {, }} \\
& \nabla_{X_{k}} Z=\nabla_{Z} X_{k}=-j \nabla_{Z} j X_{k}=\frac{1}{2}\left(\varepsilon_{k}-\varepsilon_{l}\right)\left(j X_{k}\right) j Z=\frac{1}{2} j Z, \\
& {\left[X_{l}, Z\right]=\left[j X_{l}, j Z\right]-j\left[j X_{l}, Z\right]-j\left[X_{l}, j Z\right]} \\
& =\frac{1}{2}\left(\varepsilon_{k}+\varepsilon_{l}\right)\left(j X_{l}\right) j Z-\frac{1}{2}\left(\varepsilon_{k}-\varepsilon_{l}\right)\left(j X_{l}\right) j Z=j Z, \\
& \nabla_{X_{l}} Z=\left[X_{l}, Z\right]+\nabla_{Z} X_{l}=j Z-j \nabla_{Z} j X_{l}=\frac{1}{2} j Z, \\
& {\left[X_{k}, j Z\right]=\left[X_{l}, j Z\right]=0 \text {, }} \\
& \nabla_{j Z} X_{k}=\nabla_{X_{k}} j Z=j \nabla_{X_{k}} Z=-\frac{1}{2} Z \text {, } \\
& \nabla_{j Z} X_{l}=\nabla_{X_{p}} j Z=j \nabla_{X_{l}} Z=-\frac{1}{2} Z \text {, } \\
& {[j Z, Z]=\frac{\langle j Z, j Z\rangle}{\omega\left(X_{k}\right)} X_{k}=\frac{1}{\omega\left(X_{k}\right)} X_{k} \quad(\sec (13)),} \\
& \nabla_{j z} Z=[j Z, Z]+j \nabla_{z} Z \\
& =\frac{1}{\omega\left(X_{k}\right)} X_{k}+j H_{\frac{1}{2}\left(e_{k}-\varepsilon_{l}\right)}=\frac{1}{2}\left(\frac{X_{k}}{\omega\left(X_{k}\right)}+\frac{X_{l}}{\omega\left(X_{l}\right)}\right) .
\end{aligned}
$$


More computation then gives

$$
\begin{aligned}
\nabla_{U} U=- & \frac{1}{2}(a+b) j Z+\frac{1}{2}\left(H_{\varepsilon_{k}}+H_{\varepsilon_{q}}\right) \\
\nabla_{U} V=- & \frac{1}{2}(\alpha+\beta) Z-\frac{1}{2}\left(j H_{\varepsilon_{k}}+j H_{\varepsilon}\right) \\
\nabla_{V} U=- & \frac{1}{2}(\alpha+\beta+a-b) Z+\left(\alpha a \omega\left(X_{k}\right)+\frac{1}{2}\right) j H_{\varepsilon_{k}} \\
+ & \left(\beta b \omega\left(X_{l}\right)-\frac{1}{2}\right) j H_{\varepsilon_{q}} \\
\nabla_{[U, V]} V= & \frac{1}{4}(a-b)\left((\alpha-\beta) j Z+\left(H_{\varepsilon_{k}}-H_{q_{l}}\right)\right) \\
& +\left(a \alpha+1 / \omega\left(X_{k}\right)\right)\left(\alpha \omega\left(X_{k}\right) H_{\varepsilon_{k}}+\frac{1}{2} j Z\right) \\
& +b \beta\left(\beta \omega\left(X_{l}\right) H_{\varepsilon}+\frac{1}{2} j Z\right)
\end{aligned}
$$

Specializing to the values

$$
a=-\alpha=\left(\sqrt{2 \omega\left(X_{k}\right)}\right)^{-1}, \quad b=-\beta=\left(\sqrt{2 \omega\left(X_{l}\right)}\right)^{-1}
$$

gives $\langle R(U, V) V, U\rangle=-\frac{3}{4}\left(1 / \omega\left(X_{k}\right)-1 / \omega\left(X_{l}\right)\right)$ which proves the theorem.

It is appropriate here to recall two theorems proved in [2].

THEOREM 3. Suppose $\frac{1}{2} \varepsilon_{k}, \frac{1}{2} \varepsilon_{l}, \frac{1}{2}\left(\varepsilon_{k}+\varepsilon_{l}\right)$ are all roots for some pair of indices $k<l$ and that $\operatorname{dim} \mathfrak{n}_{\left(\varepsilon_{k}+\varepsilon_{1}\right) / 2}=1$. Then for any admissible form $\omega$, there exist planes with positive sectional curvature.

THEOREM 4. The metric $\langle$, > on $S$ is Einstein if and only if

$$
\frac{1}{\omega\left(X_{k}\right)}\left\{1+\frac{1}{4} \operatorname{dim} \mathfrak{n}_{\frac{1}{2} e_{k}}+\frac{1}{2} \sum_{m \neq k} \operatorname{dim} \mathfrak{n}_{\frac{1}{2}\left(e_{k}+e_{m}\right)}\right\}
$$

is a constant independent of $k=1, \ldots, R$ and in that case $\langle$,$\rangle is homothetic$ to a Bergman metric on a homogeneous bounded domain.

REMARK. In [2], it was only explicitly proved that the Einstein condition implies the constancy of (20). However, suppose $\omega$ is an admissible form for which (20) is constant. By [8, p. 66], we can construct a canonical homogeneous Siegel domain $D$ in $C^{N}\left(C^{N}\right.$ is actually $\xi$ with the complex structure given by $j$ ) with a faithful representation of $S$ as a group of affine transformations so that $S$ acts simply transitively on $D$. $D$ has a preferred base point $p$ so we get an equivariant diffeomorphism $S \ni g \rightarrow g(p) \in D$ where the pullback of the almost complex structure on $D$ at $p$ is just $j$. The Bergman metric on $D$ then pulls back to a left-invariant Kähler-Einstein metric $\langle,\rangle^{\prime}$ on $S$ which must come from an admissible form $\omega^{\prime}$. Observe that the root space decomposition of $\xi$ does not depend on the choice of admissible form so by constancy of (20), $\omega$ is a multiple of $\omega^{\prime}$ on $j a$. Since the values on ja determine the inner product, this shows $\langle$,$\rangle is Einstein and is just a multiple of the$ pulledback Bergman metric. 
REMARK. This result shows that any homogeneous, nonflat, Kähler-Einstein metric with nonpositive sectional curvature is homothetic to a homogeneous bounded domain with the Bergman metric (cf. discussion in Introduction).

3. The symmetric case. As usual, $(\xi, j)$ will be a normal $j$-algebra with admissible form $\omega$ and other notation as before. The eventual aim of this section is to obtain some necessary conditions, analogous to Theorems 1 and 2 , for the metric $\langle$,$\rangle on S$ to be symmetric. We start, however, with no restriction on $\langle$,$\rangle .$

Take $H \in a, Z \in \mathfrak{n}_{\alpha}$. Then one has

$$
R(H, Z)=\nabla_{H} \nabla_{Z}-\nabla_{Z} \nabla_{H}-\nabla_{[H, Z]}=-\alpha(H) \nabla_{Z}
$$

Then for $Y \in \mathfrak{n}_{\beta}, \alpha \neq \beta$, we have $2 \nabla_{Y} Z=[Y, Z]-(\operatorname{ad} Y)^{t} Z-(\operatorname{ad} Z)^{t} Y$ where the terms on the right are respectively in $\mathfrak{n}_{\alpha+\beta}, \mathfrak{n}_{\alpha-\beta}, \mathfrak{n}_{\beta-\alpha}$. Thus

$$
\begin{aligned}
2 R\left(H, \nabla_{Y} Z\right)= & -(\alpha+\beta)(H) \nabla_{[Y, Z]}+(\alpha-\beta)(H) \nabla_{(\operatorname{ad~} Y)^{\prime} Z} \\
& +(\beta-\alpha)(H) \nabla_{(\operatorname{ad} Z)^{t} Y}
\end{aligned}
$$

and then

$$
\begin{aligned}
\left(\nabla_{Y} R\right) & (H, Z)=\nabla_{Y} \circ R(H, Z) \\
& -R\left(\nabla_{Y} H, Z\right)-R\left(H, \nabla_{Y} Z\right)-R(H, Z) \circ \nabla_{Y} \\
= & -\alpha(H) \nabla_{Y} \nabla_{Z}+\beta(H) R(Y, Z)+\frac{1}{2}(\alpha+\beta)(H) \nabla_{[Y, Z]} \\
& -\frac{1}{2}(\alpha-\beta)(H) \nabla_{(\operatorname{ad} Y)^{\prime} Z} \\
& -\frac{1}{2}(\beta-\alpha)(H) \nabla_{(\operatorname{ad} Z)^{t} Y}+\alpha(H) \nabla_{Z} \nabla_{Y} \\
= & (\beta-\alpha)(H)\left(R(Y, Z)+\frac{1}{2} \nabla_{[Y, Z]}+\frac{1}{2} \nabla_{(\operatorname{ad} Y)^{t} Z}-\frac{1}{2} \nabla_{(\operatorname{ad} Z)^{t} Y}\right) \\
= & (\beta-\alpha)(H)\left(\nabla_{Y} \nabla_{Z}-\nabla_{Z} \nabla_{Y}-\frac{1}{2} \nabla_{[Y, Z]}+\frac{1}{2} \nabla_{(\operatorname{ad} Y)^{\prime} Z}-\frac{1}{2} \nabla_{(\operatorname{ad} Z)^{\prime} Y}\right) .
\end{aligned}
$$

Now suppose $\nabla R=0$. We may assume $\alpha-\beta$ is not a root. Computing $\left\langle\left(\nabla_{Y} R\right)(H, Z) Z, Y\right\rangle$ from (22) gives

$0=-\left\langle\nabla_{Z} Z, \nabla_{Y} Y\right\rangle+\left\langle\nabla_{Y} Z, \nabla_{Z} Y\right\rangle-\frac{1}{2}\left\langle\nabla_{[Y, Z]} Z, Y\right\rangle-\frac{1}{2}\left\langle\nabla_{(\operatorname{ad} Z)^{\prime} Y} Z, Y\right\rangle$.

From (6), (7), (9) and orthogonality of the root spaces, we have

$$
\begin{aligned}
\left\langle\nabla_{Z} Z, \nabla_{Y} Y\right\rangle & =\langle Z, Z\rangle\langle Y, Y\rangle\left\langle H_{\alpha}, H_{\beta}\right\rangle, \\
\left\langle\nabla_{Y} Z, \nabla_{Z} Y\right\rangle & =\frac{1}{4}\left\langle[Y, Z]-(\operatorname{ad} Z)^{t} Y,[Z, Y]-(\operatorname{ad} Z)^{t} Y\right\rangle \\
& =-\frac{1}{4}\langle[Y, Z],[Y, Z]\rangle+\frac{1}{4}\left\langle(\operatorname{ad} Z)^{t} Y,(\operatorname{ad} Z)^{t} Y\right\rangle, \\
\left\langle\nabla_{[Y, Z]} Z, Y\right\rangle & =\frac{1}{2}\langle[Y, Z],[Y, Z]\rangle, \\
\left\langle\nabla_{(\operatorname{ad} Z)^{t} Y} Z, Y\right\rangle & =\frac{1}{2}\left\langle\left[(\operatorname{ad} Z)^{t} Y, Z\right], Y\right\rangle=-\frac{1}{2}\left\langle(\operatorname{ad} Z)^{t} Y,(\operatorname{ad} Z)^{t} Y\right\rangle
\end{aligned}
$$


which finally gives

$$
\begin{aligned}
\langle Z, Z\rangle\langle Y, Y\rangle & \left\langle H_{\alpha}, H_{\beta}\right\rangle \\
& =-\frac{1}{2}\langle[Y, Z],[Y, Z]\rangle+\frac{1}{2}\left\langle(\operatorname{ad} Z)^{t} Y,(\operatorname{ad} Z)^{t} Y\right\rangle .
\end{aligned}
$$

Now suppose in addition that $k<l, \alpha=\frac{1}{2}\left(\varepsilon_{k}-\varepsilon_{l}\right), \beta=\frac{1}{2}\left(\varepsilon_{k}+\varepsilon_{l}\right)$ are roots and $Y=j Z$. Then from (18), $[Y, Z]=\left(\langle Y, Y\rangle / \omega\left(X_{k}\right)\right) X_{k}$. Further, $(\operatorname{ad} Z)^{t} Y$ $\in \mathfrak{n}_{e_{l}}$ so $(\operatorname{ad} Z)^{t} Y=r X_{l}$ and $r\left\langle X_{l}, X_{l}\right\rangle=\left\langle(\operatorname{ad} Z)^{t} Y, X_{l}\right\rangle=\left\langle Y,\left[Z, X_{l}\right]\right\rangle=$ - $\langle Y, j Z\rangle=-\langle Y, Y\rangle$ where the bracket is computed from (15). Thus

$$
(\operatorname{ad} Z)^{t} Y=-\left(\langle Y, Y\rangle / \omega\left(X_{l}\right)\right) X_{l} \text {. }
$$

Substituting these last results and (3), (5) into (23) gives

$$
\langle Y, Y\rangle^{2}\left\{\omega\left(X_{k}\right)^{-1}-\omega\left(X_{l}\right)^{-1}\right\}=0 \text {. }
$$

This proves the following.

THEOREM 5. Suppose $\frac{1}{2}\left(\varepsilon_{k}+\varepsilon_{l}\right), k<l$, is a root and the metric $\langle$,$\rangle on S$ is symmetric (i.e. $\nabla R=0)$. Then $\omega\left(X_{k}\right)=\omega\left(X_{l}\right)$.

REMARK. Of course when the metric is irreducible symmetric, there are also strong restrictions on the possible dimensions of the various root spaces which can be read off from the Iwasawa decompositions.

4. Examples. In this section, we will construct a family of examples to which we can apply Theorem 1 . Take a real vector space $\Xi$ of dimension $6+2 p$ having an almost complex structure $j$. In $\xi$, fix a $2 p$ (real) dimensional $j$-invariant subspace $Z$ and a basis $\left\{j r_{1}, j r_{2}, r_{1}, r_{2}, x, j x\right\}$ of a vector space complement of $Z$. Let $h$ be any real-valued $j$-invariant positive definite symmetric bilinear form on $Z$. Define a bracket product on $\xi$ so that the only nonzero terms involving basis elements or elements of $Z$ satisfy

$$
\left[j r_{1}, r_{1}\right]=r_{1}, \quad\left[j r_{1}, x\right]=\frac{1}{2} x, \quad\left[j r_{1}, j x\right]=\frac{1}{2} j x, \quad\left[j r_{1}, z\right]=\frac{1}{2} z
$$

for all $z \in Z$,

$$
\begin{aligned}
{\left[j r_{2}, x\right] } & =-\frac{1}{2} x, \quad\left[j r_{2}, j x\right]=\frac{1}{2} j x, \quad\left[j r_{2}, r_{2}\right]=r_{2}, \\
{\left[r_{2}, x\right] } & =j x, \quad[x, j x]=-r_{1}, \quad\left[z_{1}, z_{2}\right]=h\left(z_{1}, j z_{2}\right) r_{1} \quad \text { for } z_{1}, z_{2} \in Z .
\end{aligned}
$$

Of course this bracket is to be skew-symmetric and bilinear. It is easy to verify this determines a Lie algebra structure on $\xi, \mathfrak{s}$ is solvable, $\mathfrak{n}=[\xi, \mathfrak{\xi}]=$ $\operatorname{span}\left\{r_{1}, r_{2}, x, j x, Z\right\}$, and an abelian complementary space is $a=$ $\operatorname{span}\left\{j r_{1}, j r_{2}\right\}$. Further one checks condition (D2) relating $j$ and the bracket. Now we want to define $\omega$ so as to satisfy the remaining conditions. Condition (D3) implies $\omega(x)=\omega(j x)=\omega(Z)=0$ and the values of $\omega$ on $j r_{1} j r_{2}$ are irrelevant and may also be taken to be zero. Finally, we can normalize so that $\omega\left(r_{1}\right)=1, \omega\left(r_{2}\right)=t>0$. It is easy to check that $\omega$ defined in this way does satisfy conditions (D1)-(D5), and our previous comments imply that, up to 
constant multiple, we get all inner products $\langle$,$\rangle arising from admissible \omega$ in this way.

Let $\varepsilon_{k}$ be the linear functional on a defined by $\varepsilon_{k}\left(j r_{m}\right)=\delta_{k m}$. Then the roots and root spaces are easily found to be

$$
\begin{aligned}
\mathfrak{n}_{e_{1}}=\mathbf{R} r_{1}, \quad \mathfrak{n}_{e_{2}} & =\mathbf{R} r_{2}, & \mathfrak{n}_{\frac{1}{2} e_{1}}=Z, \quad \mathfrak{n}_{\frac{1}{2} e_{2}}=0, \\
\mathfrak{n}_{\frac{1}{2}\left(e_{1}-\varepsilon_{2}\right)} & =\mathbf{R} x, & \mathfrak{n}_{\frac{1}{2}\left(e_{1}+e_{2}\right)}=\mathbf{R} j x .
\end{aligned}
$$

Also note $j r_{1}, j r_{2}, r_{1}, r_{2}, x, j x$ are pairwise orthogonal and orthogonal to $Z$ and $\left\langle r_{1}, r_{1}\right\rangle=\langle x, x\rangle=1,\left\langle r_{2}, r_{2}\right\rangle=t, h\left(z_{1}, z_{2}\right)=\left\langle z_{1}, z_{2}\right\rangle,\langle$,$\rangle is j$-invariant.

Note the case $p=1$ just gives the original Pyatetskii-Shapiro example of a homogeneous nonsymmetric domain.

Now by Theorem $4,\langle$,$\rangle is Einstein if and only if t=3 /(3+p)$. These values then give a multiple of the Bergman metric on a homogeneous domain. However $\omega\left(r_{1}\right)>2 \omega\left(r_{2}\right)$ for $p>3$ so by Theorem 1 , these Bergman metrics have some positive holomorphic sectional curvatures.

It is interesting to contrast the situation for $p=1, t=\frac{3}{4}$. Here Theorem 2 shows there is some positive sectional curvature but a tedious calculation shows that for

$$
U=u_{1} j r_{1}+u_{2} j r_{2}+u_{3} r_{1}+u_{4} r_{2}+u_{5} x+u_{6} j x+z
$$

we have

$$
\begin{aligned}
4\langle R(U, j U) j U, & U\rangle=-4\left(u_{1} u_{5}-u_{3} u_{6}-u_{4} u_{6}+u_{2} u_{5}\right)^{2} \\
& -4\left(-u_{1} u_{6}-u_{3} u_{5}+u_{4} u_{5}+u_{2} u_{6}\right)^{2} \\
& -4\left|u_{5} j z+u_{6} z\right|^{2}-4\langle z, z\rangle^{2} \\
& -4\langle z, z\rangle\left(2 u_{1}^{2}+2 u_{3}^{2}+u_{5}^{2}+u_{6}^{2}\right)-4\left(u_{1}^{2}+u_{3}^{2}\right)^{2} \\
& -4\left(u_{1}^{2}+u_{3}^{2}\right)\left(u_{5}^{2}+u_{6}^{2}\right)-\left(\sqrt{3}\left(u_{2}^{2}+u_{4}^{2}\right)+2\left(u_{5}^{2}+u_{6}^{2}\right) / \sqrt{3}\right)^{2}
\end{aligned}
$$

which is easily seen to be (strictly) negative unless $U=0$ (see [2] for a similar calculation which also gives a 1-parameter deformation of Kähler metrics leading from this Bergman metric to a metric of nonpositive sectional curvature). Thus, we have proved the following.

THEOREM 6. There are bounded homogeneous domains of every complex dimension $>6$ which in the Bergman metric have some positive holomorphic sectional curvature. There is also a bounded homogeneous domain of complex dimension 4 (the original nonsymmetric example of Pyatetskii-Shapiro) which in the Bergman metric has some positive sectional curvature but whose holomorphic sectional curvature is strictly negative. 


\section{BIBLIOGRAPHY}

1. R. Azencott and E. N. Wilson, Homogeneous manifolds with negative curvature. I, Trans. Amer. Math. Soc. 215 (1976), 323-362.

2. J. E. D'Atri, The curoature of homogeneous Siegel domains, J. Differential Geometry (to appear).

3. S. G. Gindikin, I. I. Pjateckii-Shapiro and E. B. Vinberg, Homogeneous Kähler manifolds, Geometry of Homogeneous Bounded Domains, C.I.M.E. 1967, 1-88.

4. S. Kobayashi and K. Nomizu, On automorphisms of a Kaehlerian structure, Nagoya Math. J. 11 (1957), 115-124.

5. S. Kobayashi, Homogeneous Riemannian manifolds of negative curvature, Tôhoku Math. J. 14 (1962), 413-415.

6. J. L. Koszul, Sur la forme hermitienne canonique des expaces homogenes complexes, Canad. J. Math. 7 (1955), 562-576.

7. S. Murakami, On automorphisms of Siegel domains, Lecture Notes in Math., vol. 286, Springer-Verlag, Berlin and New York, 1971.

8. I. I. Pyatetskii-Shapiro, Automorphic functions and the geometry of classical domains, English transl., Gordon and Breach, New York, 1969.

9. H. Rossi, mimeographed lecture notes, Brandeis University.

10. H. Rossi and M. Vergne, Representations of certain soloable Lie groups on Hilbert spaces of holomorphic functions and applications to the holomorphic discrete series of a semisimple Lie group, J. Functional Analysis 13 (1973), 324-389.

11. E. B. Vinberg, S. G. Gindikin and I. I. Pjateckii-Shapiro, Classification and canonical realization of complex bounded homogeneous domains, Trudy Moskov. Mat. Obšc. 12 (1963), 359-388, English transl., Trans. Moscow Math. Soc. 12 (1963), 404-437, Amer. Math. Soc., Providence, R.I., 1965.

Departmant of Mathematics, Rutgers Universtiy, New Brunswick, New Jersey 08903 